\title{
Attitudes to Ageing and Change in Frailty Status: The English Longitudinal Study of Ageing
}

\author{
Catharine R. Gale ${ }^{a, b}$ Cyrus Cooper ${ }^{a}$ \\ ${ }^{a}$ MRC Lifecourse Epidemiology Unit, University of Southampton, Southampton, and ${ }^{b}$ Centre for Cognitive Ageing \\ and Cognitive Epidemiology, Department of Psychology, University of Edinburgh, Edinburgh, UK
}

\section{Keywords}

Frailty · Attitudes to ageing $\cdot$ Cohort $\cdot$ Longitudinal study

\begin{abstract}
Background: Older people with more negative attitudes to ageing are at increased risk of several adverse outcomes, including decline in physical function and increased difficulties with activities of daily living. Objective: We investigated whether negative attitudes to ageing increase the risk of the onset or progression of frailty. Method: Participants were 3,505 men and women aged 60 years and over from the English Longitudinal Study of Ageing. They completed a 12item questionnaire on attitudes to ageing. Exploratory factor analysis was used to examine the structure of these items, and a single factor was derived which we labelled "physical and psychological loss." Frailty was assessed by the Fried phenotype of physical frailty at waves 2 and 4 , and by a frailty index at waves 2-5. Results: Having a more positive attitude to ageing as regards "physical and psychological loss" was associated with a decreased risk of becoming physically frail or pre-frail at follow-up. For a standard deviation increment in score, the relative risk ratios (95\% confidence interval), adjusted for age, sex and baseline level of physical frailty, were $0.86(0.79,0.94)$ for pre-frailty and $0.72(0.63,0.83)$
\end{abstract}

\section{KARGER}

E-Mail karger@karger.com www.karger.com/ger

This article is licensed under the Creative Commons Attribution 4.0

\section{(9) 2017 The Author(s) \\ Published by S. Karger AG, Basel \\ Karger \\ Open access} International License (CC BY) (http://www.karger.com/Services/ OpenAccessLicense). Usage, derivative works and distribution are permitted provided that proper credit is given to the author and the original publisher. for frailty. Further adjustment for other potential confounding variables had only slight attenuating effects on these associations: multivariable-adjusted relative risk ratios were $0.89(0.81,0.98)$ for pre-frailty and $0.78(0.68,0.91)$ for frailty. Attitude to ageing was not associated with change in the frailty index over time after adjustment for potential confounding variables. Conclusion: Older people who have a more positive attitude to ageing are at reduced risk of becoming physically frail or pre-frail. Future research needs to replicate this finding and discover the underlying mechanisms. Attitude to ageing was not a risk factor for change in the more broadly defined frailty index.

(c) 2017 The Author(s)

Published by S. Karger AG, Basel

\section{Introduction}

How older people view ageing - based on their expectations or their own experiences - may have implications for their later mental and physical health. Longitudinal studies have shown that older people who have a more negative perception of ageing - seeing it as a time of increasing ill health, social isolation, loss of ability to perform usual activities and loss of independence - tend to experience an increase in depressive symptoms [1] and

Prof. Catharine R. Gale

MRC Lifecourse Epidemiology Unit

Southampton General Hospital

Southampton SO16 6YD (UK)

E-Mailcrg@mrc.soton.ac.uk 
have a higher risk of incident anxiety or depression [2]. Having a more negative view of ageing has also been associated prospectively with declines in cognitive ability [3], in objectively measured physical function $[4,5]$, in self-reported functional health [6] and in self-rated health [7] as well as an increased risk of the onset of difficulties in activities of daily living [8] and a higher mortality [9]. The potential influence of such attitudes may extend over many years: in the Baltimore Longitudinal Study of Ageing, participants holding more negative views of ageing had a steeper decline in hippocampal volume and a greater accumulation of other Alzheimer disease biomarkers - amyloid plaques and neurofibrillary tangles - more than 20 years later [10].

Frailty is a clinical syndrome that becomes increasingly common at older ages. It is characterised by heightened vulnerability to stressors due to lowered physiological reserves, decline in the ability to maintain homeostasis and impairments in multiple systems. The 2 principal models of frailty are the Fried phenotype model, in which frailty is defined on purely physical terms, based on 3 or more components (poor grip strength, slow walking speed, low physical activity, exhaustion and unintentional weight loss) [11], and the frailty index, or cumulative deficit model, in which frailty is defined much more broadly in terms of the accumulation of "deficits" (symptoms, signs, diseases and disabilities) [12]. Psychological factors may play a part in determining the risk of frailty. Lower psychological wellbeing [13], increased depression [14] and poorer cognitive ability [15] have all been associated with higher risk of incident physical frailty, as defined by the Fried phenotype. It is common practice to count depressive symptoms and poorer cognitive ability among the deficits that make up a frailty index. In some longitudinal studies where frailty indices have been derived without these psychological factors, there is evidence to link depression with worsening frailty status [16].

To our knowledge, there is no longitudinal evidence on whether having a more negative attitude to ageing puts older people at greater risk of becoming frail. In view of the prospective evidence linking negative attitudes to ageing with greater decline in objectively measured physical function $[4,5]$, increased difficulties in self-reported physical function and in activities of daily living $[6,8]$ and worse self-rated health [7], we hypothesised that older people with more negative attitudes to ageing would be at higher risk of the onset or progression of frailty. We used data from the English Longitudinal Study of Ageing (ELSA) to investigate this relationship. We characterised frailty using both the Fried phenotype and a frailty index in order to assess whether relationships between attitudes to ageing and change in frailty status were consistent regardless of how frailty was defined.

\section{Methods}

\section{Participants}

The initial sample for ELSA was based on people aged $\geq 50$ years who had participated in the Health Survey for England in 1998, 1999 or 2001. It was drawn by postcode sector and stratified by health authority and proportion of households in non-manual socioeconomic groups. The initial survey took place in 2002-2003. Subsequent waves of data collection have taken place at 2-year intervals. Refreshment samples drawn from the Health Survey for England were added at wave 3 and 4 to maintain the representation of people aged 50-75 years. The current study uses data from waves 2 (2004-2005), 3 (2006-2007), 4 (2008-2009) and 5 (20102011). At waves 2 and 4 , core sample members (those in the household aged $\geq 50$ years who had taken part in wave 1 ) who had completed the main interview were invited to have a visit from a nurse that included measurements of physical function.

\section{Measures}

Attitudes to Ageing

At wave 2, participants completed a 12-item questionnaire on attitudes to ageing. Items were derived from respondents' answers to 2 open-ended questions in the pilot study for wave 2: "What would you say are the most positive things about growing older?" and "What would you say are the most negative things about growing older?" Before completing the questionnaire, participants were asked: "Thinking of old age and your own ageing experience, to what extent do you agree or disagree with the following statements." There were 5 response options, ranging from "strongly agree" to "strongly disagree." The 12 items are shown in Table 1. To our knowledge, no paper has been published on the psychometrics of this scale. We carried out an exploratory factor analysis to explore the structure of the attitude items. Analyses were based on 7,974 participants. Both the scree slope and the eigenvalues (the number $>1$ ) from the initial principal components analysis suggested a model with 3 components, explaining $45.8 \%$ of the total variance. The varimax-rotated components were inspected for items that had salient loadings $(\geq 0.5)$. We refer to the rotated components as factors. We labelled factor 1 "physical and psychological loss." Four items had high positive loadings on this factor. They included: "I expect to become more lonely with age" (0.74), "Old age is a time of ill-health" (0.73), "Old age is a time of loneliness" $(0.79)$ and "I worry my health will get worse as I grow older" (0.63). The Cronbach $\alpha$ for these items was 0.76 . Factor 2 had 4 highloading items, but these were less coherent, reflecting in part attitudes to physical change but also to psychosocial advantages. These items were: "I don't think of myself as old," "Growing old doesn't bother me," "As I get older, I expect to be able to do the things I've always done" and "Retirement is a time of leisure." The Cronbach $a$ for these items was 0.47 . Factor 3 had only 2 highloading items: "As I grow older, I become more tolerant" and "We can learn a lot from old people." The Cronbach a for these items was 0.29 . Factor 2 and 3 were not considered further because of 
Table 1. Attitude to ageing items

\begin{tabular}{|c|c|}
\hline 1 & We can learn a lot from old people \\
\hline 2 & As I get older, I expect to become more lonely \\
\hline 3 & Old age is a time of ill health \\
\hline 4 & As I grow older, I become more tolerant \\
\hline 5 & Old age is a time of loneliness \\
\hline 6 & $\begin{array}{l}\text { As I get older, I expect to be able to do the things I've } \\
\text { always done }\end{array}$ \\
\hline 7 & $\begin{array}{l}\text { When I think of old people, I think of them as generally } \\
\text { grumpy and miserable }\end{array}$ \\
\hline 8 & I worry that my health will get worse as I grow older \\
\hline 9 & I don't think of myself as old \\
\hline 10 & Old people don't get respect in society \\
\hline 11 & Retirement is a time of leisure \\
\hline 12 & Growing old doesn't bother me \\
\hline
\end{tabular}

their lack of internal consistency. Factor composite scores were calculated for "physical and psychological loss" such that higher scores indicated a more positive attitude to ageing.

\section{Frailty}

Data from waves 2 and 4 were used to derive the Fried phenotype of physical frailty [11]. These criteria define frailty as the presence of 3 or more of the following: unintentional weight loss, weakness, self-reported exhaustion, slow gait speed and low physical activity. The presence of 1 or 2 of these criteria defines pre-frailty. Height and weight were measured with a portable stadiometer and electronic scales, respectively. Body mass index (BMI) was calculated as weight (in kilograms)/height (in metres) ${ }^{2}$. Maximum handgrip strength was measured 3 times on each side using a dynamometer; the best of these measurements was used for analysis. Gait speed was assessed in participants aged 60 years and over by measuring the time taken to walk a distance of 8 feet at usual pace; the timed walk was repeated, and the mean of the 2 measurements was calculated. Participants responded to 3 questions about the frequency with which they did vigorous, moderate or mild exercise. We ranked the combinations of responses to these questions according to the amount and intensity of exercise involved to provide an estimate of usual physical activity. Participants completed the 8-item Center for Epidemiologic Studies Depression Scale (CES-D) [17]. We operationalized the Fried phenotype criteria using definitions very similar to those used in the original phenotype of frailty studies $[11,18]$ : weight loss was defined as either loss of $\geq 10 \%$ of body weight since the initial survey (for frailty at wave 2 ) or since wave 2 (for frailty at wave 4 ) or current BMI $<18.5$; weakness was defined as maximum grip strength in the lowest $20 \%$ of the distribution, after taking sex and BMI into account; exhaustion was considered present if the participant gave a positive response to either of the CES-D items "Felt that everything I did was an effort in the last week" or "Could not get going in the last week"; slow walking speed was defined as a walking speed in the lowest $20 \%$ of the distribution, after taking account of sex and height; and low physical activity was defined as physical activity in the lowest sexspecific $20 \%$ of the distribution.

Data from waves 2, 3, 4 and 5 were used to derive a frailty index. A frailty index can be derived from different numbers or types of variables, thereby facilitating comparison between datasets [12], although it is recommended that at least 30 deficits are included to ensure that estimates are precise [19]. The criteria for inclusion are that the variables are associated with health status, represent conditions that become more common with age - though not ubiquitous (e.g., presbyopia) - and cover a range of systems [19]. Our frailty index was made up of 52 deficits, including sensory and functional impairments, a score on a composite measure of cognitive function and self-reported co-morbidities (see Appendix). The frailty index is constructed by summing the number of deficits present for each individual and dividing by the total number of deficits considered, which gives a range from 0 to 1 . Higher values indicate greater frailty.

\section{Covariates}

We chose age, socioeconomic position, educational qualifications, smoking status and depressive symptoms at baseline as potential confounding variables. In models where change in the Fried phenotype of frailty was the outcome, we also adjusted for number of chronic physical diseases and number of components of the phenotype present at baseline. (Diagnoses of disease were among the "deficits" used to derive the frailty index, so it would have been inappropriate to include them as covariates in models where the frailty index was the outcome.)

Socioeconomic position was indexed by total household wealth, including savings and investments, value of any property or business assets and net of debt, excluding pension assets. Household wealth has been identified as the most accurate indicator of longterm socioeconomic circumstances in ELSA [20]. Participants were asked about their educational qualifications. The highest educational qualification obtained was classified into 7 categories; we reversecoded these so that higher categories indicated greater educational attainment ( 7 = national vocational qualification level 4 [NVQ4]/ NVQ5/degree or equivalent; 6 = higher education below degree; $5=\mathrm{NVQ} 3 /$ General Certificate of Education [GCE] A level equivalent; 4 = NVQ2/GCE O level equivalent; 3 = NVQ1/Certificate of Secondary Education [CSE] other grade equivalent; 2 = foreign/ other; and $1=$ no qualification). Participants were asked whether a doctor had ever told them that they had any of the following conditions: high blood pressure/hypertension, angina, heart attack, congestive heart failure, diabetes or high blood sugar, a stroke, chronic lung disease, asthma, arthritis or rheumatism, osteoporosis or cancer. We used this information to calculate the number of chronic physical diseases present. Symptoms of depression were assessed using the 8-item version of the CES-D [17]. As 2 items from the scale were used as an indicator of exhaustion when deriving the physical frailty phenotype, we calculated a total CES-D score after excluding these items for use as a covariate in the analyses of attitudes to ageing in relation to change in physical frailty. A total CES-D score based on all 8 items was used for all other analyses.

\section{Statistical Analysis}

In total, 6,183 core cohort members aged $\geq 60$ years participated in wave 2 . Of those, 3,505 were re-interviewed at waves 3,4 and 5 and had sufficient data (on at least 30 out of a potential 52 variables) to allow the derivation of a frailty index at each wave; 3,243 (52\%) were re-interviewed and re-assessed by a nurse at wave 4 and had data on physical frailty at that wave. In total, 3,017 of those with complete frailty index data and 2,505 of those with complete physical frailty data had data on all other variables of interest. 
Table 2. Baseline characteristics of the participants and their rank order correlations with the attitudes to ageing score on "physical and psychological loss" $(n=3,505)$

\begin{tabular}{lcc}
\hline Characteristics & $\begin{array}{l}\text { Mean (SD), } \\
\text { median (IQR) or } \\
(\%)\end{array}$ & $\begin{array}{c}\text { Correlation with } \\
\text { "physical and } \\
\text { psychological loss" }\end{array}$ \\
\hline Mean age (SD), years & $69.8(7.09)$ & -0.005 \\
Female, $n$ (\%) & $2,005(57.2)$ & -0.003 \\
Median household wealth (IQR), GBP & $204,500(109,082-350,850)$ & $0.075^{* *}$ \\
Educational qualifications, $n$ (\%) & & $0.063^{* *}$ \\
$\quad$ No qualifications & $1,335(38.1)$ & \\
Foreign/other & $351(10.0)$ & \\
$\quad$ NVQ1/CSE other grade equivalent & $163(4.65)$ & \\
$\quad$ NVQ2/GCE O level equivalent & $586(16.7)$ & $-0.140^{* * *}$ \\
NVQ3/GCE A level equivalent & $190(5.42)$ & $-0.040^{*}$ \\
Higher education below degree & $447(12.8)$ & $-0.324^{* * *}$ \\
$\quad$ NVQ4/NVQ5/degree or equivalent & $431(12.3)$ & $-0.141^{* * *}$ \\
Median number of chronic physical illnesses (IQR) & $1(0-2)$ & $-0.228^{* * *}$ \\
Current smoker, $n$ (\%) & $398(11.4)$ & - \\
Median depressive symptoms (IQR) & $1(0-2)$ & $0(0-1)$ \\
Median number of components of frailty present (IQR) ${ }^{\mathrm{a}}$ & $0.15(0.11-0.23)$ & \\
Median frailty index score (IQR) & $13.9(4.00)$ & \\
Mean “physical and psychological loss" (SD) & & \\
\hline
\end{tabular}

SD, standard deviation; IQR, interquartile range; NVQ, national vocational qualification level; CSE, Certificate of Secondary Education; GCE, General Certificate of Education. ${ }^{a}$ Descriptive data on the Fried phenotype of frailty are based on 3,243 participants. ${ }^{* * *} p<0.001,{ }^{* *} p<0.01,{ }^{*} p<0.05$.

Missingness in variables of interest was significantly correlated with other measured variables, which is consistent with the requirements for imputing missing at random data [21]. We therefore used multiple multivariate imputation to impute values for baseline predictors and covariates with missing values using IBM SPSS Statistics 21 software. In total, 16\% of participants with complete frailty index data and $23 \%$ of participants with complete physical frailty data had some missing data on predictors or covariates. We generated 23 imputation datasets using chained equation imputation [22]. The pooled effect sizes from analysis with imputed data were very similar but slightly stronger than those obtained from analysis using the sample with complete data, suggesting possible bias in the latter sample. We present results from analyses based on imputation.

We used rank order correlations to examine attitudes to ageing in relation to the other baseline characteristics. We used linear regression to calculate change in frailty index score between waves 2 and 5 according to attitude to "physical and psychological loss" at baseline. We used multinomial logistic regression to calculate relative risk ratios for the onset of physical frailty or pre-frailty at wave 4, according to attitude to "physical and psychological loss" at the wave 2 baseline. The association between attitude to "physical and psychological loss" and the 2 frailty outcomes did not differ by sex, so we analysed men and women together and adjusted for sex. Estimates are shown adjusted first for age and sex and then further adjusted for the other covariates. All estimates were weighted using longitudinal weights supplied with the data to minimize bias due to attrition since wave 1 .

Attitudes to Ageing and Change in Frailty Status
Statement of Ethics

Ethical approval was obtained from the Multicentre Research and Ethics Committee. Participants gave written informed consent.

\section{Results}

Table 2 shows the baseline characteristics of the sample and how those characteristics correlated with scores on the attitudes to ageing factor "physical and psychological loss." Having a more negative attitude to ageing was associated with lower household wealth, lower educational attainment, being physically frailer (on both measures of frailty), having more chronic physical diseases, being more depressed and being a current smoker.

At baseline, of the people with data on physical frailty, $5.6 \%$ were physically frail and $38.3 \%$ were pre-frail. By the time of the wave 4 follow-up, around 4 years later, $11.9 \%$ of them were frail and $41.3 \%$ were pre-frail. Overall change in the frailty index from baseline to wave 5 , around 6 years later, was slight, with the median (interquartile range) score changing from $0.15(0.11-0.23)$ at baseline to $0.15(0.10-0.24)$ at wave 5 , although there was consid- 
Table 3. RRR (95\% CI) for becoming physically frail or pre-frail at follow-up according to attitudes to ageing score for "physical and psychological loss" at baseline $(n=3,243)$

\begin{tabular}{|c|c|c|c|c|}
\hline & \multicolumn{2}{|c|}{$\begin{array}{l}\text { RRR ( } 95 \% \text { CI), adjusted for age, sex } \\
\text { and number of components of } \\
\text { frailty present at baseline }\end{array}$} & \multicolumn{2}{|c|}{$\begin{array}{l}\text { RRR (95\% CI), further adjusted for } \\
\text { education, household wealth, } \\
\text { depressive symptoms, chronic physical } \\
\text { illness and smoking status } \\
\text { at baseline }\end{array}$} \\
\hline & pre-frail & frail & pre-frail & frail \\
\hline $\begin{array}{l}\text { "Physical and psychological } \\
\text { loss," per SD }\end{array}$ & $0.86(0.79,0.94)$ & $0.72(0.63,0.83)$ & $0.89(0.81,0.98)$ & $0.78(0.68,0.91)$ \\
\hline
\end{tabular}

RRR obtained from multinomial logistic regression models. All estimates are weighted to reduce potential bias due to attrition. RRR, relative risk ratios; CI, confidence interval; SD, standard deviation.

Table 4. Regression coefficients (95\% CI) for change in frailty index (per SD) by wave 5 according to attitudes to ageing score for "physical and psychological loss" at baseline $(n=3,505)$

\begin{tabular}{|c|c|c|}
\hline & $\begin{array}{l}\text { Regression coefficient } \\
\text { (95\% CI), adjusted for } \\
\text { age and sex }\end{array}$ & $\begin{array}{l}\text { Regression coefficient ( } 95 \% \mathrm{CI}) \text {, further adjusted } \\
\text { for education, household wealth, depressive } \\
\text { symptoms and smoking status at baseline }\end{array}$ \\
\hline $\begin{array}{l}\text { "Physical and psychological } \\
\text { loss," per SD }\end{array}$ & $0.045(0.009,0.081)$ & $0.030(-0.080,0.069)$ \\
\hline
\end{tabular}

erable individual variation in the extent of change. The correlation at baseline between physical frailty status and the frailty index was moderate in size $(\rho=0.42)$.

Table 3 shows relative risk ratios $(95 \%$ confidence intervals $[\mathrm{CI}]$ ) for becoming physically frail or pre-frail at wave 4 , given level of frailty at baseline, according to attitude to ageing score for "physical and psychological loss" at baseline. In initial models, adjusted for age, sex and number of components of the phenotype present at baseline, participants with a more positive attitude to ageing had a significantly reduced risk of becoming physically frail or pre-frail by the time of follow-up around 4 years later: for a standard deviation (SD) increment in score, relative risk ratios $(95 \% \mathrm{CI})$ were $0.86(0.79,0.94)$ for pre-frailty and $0.72(0.63,0.83)$ for frailty. After further adjustment for household wealth, education, depressive symptoms, smoking status and number of chronic physical illnesses, these associations were only slightly attenuated: multivariable-adjusted relative risk ratios $(95 \%$ CI) were $0.89(0.81,0.98)$ for pre-frailty and $0.78(0.68$,
$0.91)$ for frailty. When we repeated these analyses adjusting for change in depressive symptoms and in number of chronic physical illnesses between baseline and follow-up in place of baseline levels of these covariates, effect sizes were slightly larger: $0.86(0.78,0.94)$ for pre-frailty and $0.71(0.62,0.82)$ for frailty.

Table 4 shows regression coefficients (95\% CI) for change in the frailty index from baseline to wave 5 , around 6 years later, according to attitude to ageing score for "physical and psychological loss" at baseline. In the initial model adjusted for age and sex, a more positive attitude to ageing was associated with an increase in the frailty index over time: for an SD increment in score, the frailty index increased by 0.045 of an SD (95\% CI 0.009, 0.081). However, this association was attenuated and no longer significant after further adjustment for household wealth, educational attainment, depressive symptoms and smoking status: for an SD increment in score, the multivariable-adjusted regression coefficient for frailty index change was $0.030(-0.080,0.069)$. 


\section{Discussion}

In this population-based sample of people aged 60 years and over, having a more positive attitude to ageing was associated with a reduced risk of becoming physically frail or pre-frail during the follow-up period. Adjustment for potential confounding factors only slightly attenuated these associations. Having a more positive attitude to ageing was not associated with the rate of change in a frailty index over the follow-up period after adjustment for potential confounding factors.

Previous longitudinal evidence has shown that having a more negative attitude to ageing is a risk factor for decline in physical function $[4,5]$ and for increased difficulty with activities of daily living $[6,8]$. Results from an intervention study in older people suggest that subliminal exposure to positive age stereotypes strengthens positive self-perceptions of ageing and leads to improved physical function [23]. Our finding that risk of onset of physical frailty or pre-frailty is reduced in those with a more positive view of ageing is consistent with these earlier observations. It also raises the possibility that interventions to modify age stereotypes may help prevent physical frailty in later life.

Although having a more positive attitude to ageing was associated with a lower risk of becoming physically frail, as defined by the Fried phenotype, we found no evidence to indicate that such an attitude might be protective as regards change in the frailty index. In an initial ageand sex-adjusted analysis, having a more positive attitude was associated with a greater increase in the frailty index over the follow-up period, though this association did not survive adjustment for other potential confounding factors. The reason for this discrepancy in our findings as regards the potential role of attitudes to ageing in influencing future frailty risk is uncertain. One explanation may lie in the nature of the 2 measures of frailty. While physical frailty is a specific medical syndrome, "characterised by diminished strength, endurance, and reduced physiologic function" [24], the cumulative deficit or frailty index model views frailty as a much wider construct, involving impairments in multiple systems. According to a consensus conference on frailty, physical frailty should be distinguished from this broader definition of frailty, which describes the general condition of an individual [24]. It is worth noting that in the current study, as has been shown previously [25], the correlation between the physical frailty measure (the phenotype of frailty) and the frailty index was moderate in size. If the adverse effect of negative attitudes to ageing is primarily on physical func-

Attitudes to Ageing and Change in Frailty Status tion $[4,5,23]$, then perhaps it is unsurprising that in this study attitudes to ageing were associated with risk of physical frailty but not with change in the more broadly defined frailty index. Although there is some longitudinal evidence linking negative attitudes to ageing in older people with some of the "deficits" included in our frailty index - namely, difficulties with activities of daily life [8], poorer self-rated health [7] and decline in cognitive ability [3] - the index also included a large number of other "deficits," among them diagnoses of a range of disorders, sensory impairments and pain. Most longitudinal studies of risk factors for frailty tend to use a single measure of frailty. Our findings indicate that the 2 most widely used models of frailty do not necessarily have the same risk factors.

The mechanisms underlying our findings linking attitudes to ageing with physical frailty risk are unclear. One potential mechanism is health behaviour. There is some evidence that older people with more positive attitudes to ageing tend to behave in a healthier fashion as regards smoking, physical activity and diet [7, 26, 27]. Here, we found that the likelihood of being a current smoker was less in those with a more positive attitude to ageing, but adjustment for smoking status had little effect on the association between attitudes to ageing and risk of physical frailty. As low physical activity is a component of the Fried frailty phenotype, and we had no data on activity from earlier in life, we were not able to explore the extent to which physical activity might account for the association between attitudes to ageing and risk of physical frailty. Future research should investigate this, given the evidence linking mid-life physical activity with later physical frailty risk [28].

The strengths of our study include the large sample size and the fact that ELSA is designed to be representative of the community-dwelling English population aged 50 years and over. One important limitation is that the psychometric properties of the attitudes to ageing scale used in ELSA were poor. Our exploratory factor analysis showed that of the 12 items included in the scale, only 4 made up a coherent factor with good internal consistency. Scores on this factor - which we labelled "physical and psychological loss" - were strongly associated with risk of becoming physically frail or pre-frail, but they represent just 1 potential domain of attitudes to ageing. Some other measures for assessing attitudes to ageing include subscales on several domains $[29,30]$. Another limitation is that due to attrition our analyses were based on $52 \%$ of the participants assessed at baseline. However, all estimates have been weighted to take account of differential 
loss to follow-up. To avoid potential bias due to the exclusion of those with missing data, we used multiple multivariate imputation to impute values for predictors and covariates with missing data. Pooled estimates based on imputed data were very similar to and slightly stronger than those based on complete data.

Negative stereotypes about ageing are common [31]. Understanding the extent to which such internalised stereotypes affect health outcomes in older people has particular relevance in our rapidly ageing populations. In this nationally representative sample, we found that older people who had a more negative attitude to ageing were at increased risk of becoming physically frail or pre-frail. Future research needs to replicate this finding and discover the underlying mechanisms.

\section{Acknowledgments}

The data were made available through the UK Data Archive. ELSA was developed by a team of researchers based at the National Centre for Social Research, University College London and the Institute for Fiscal Studies. The data were collected by the National Centre for Social Research. The funding is provided by the National Institute of Aging in the United States and a consortium of UK government departments coordinated by the Office for National Statistics. The developers and funders of ELSA and the Archive do not bear any responsibility for the analyses or interpretations presented here. This work was supported by the Medical Research Council (MRC_MC_ UU_12011/2 and MRC_MC_UP_A620_1015).

\section{Disclosure Statement}

There are no conflicts of interest to declare.

\section{Appendix 1}

Deficits Included in the Frailty Index and How They Were Scored

\begin{tabular}{|c|c|}
\hline Type of deficit & Deficit \\
\hline Problems with ... & 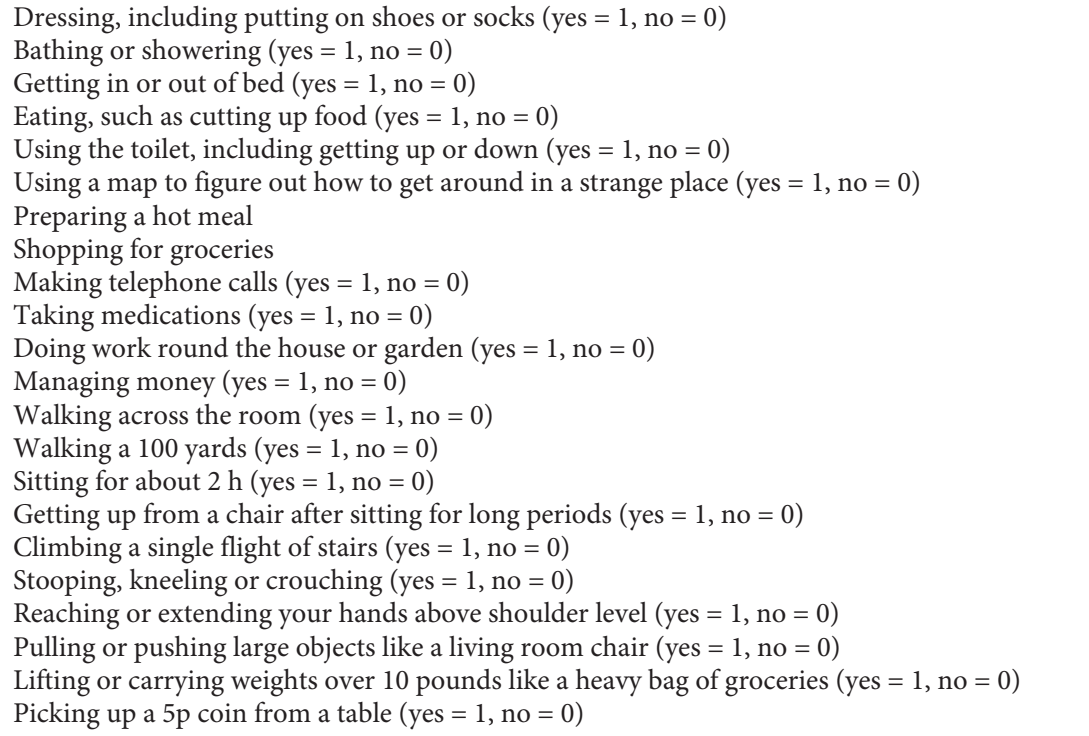 \\
\hline Doctor has diagnosed: & $\begin{array}{l}\text { Angina }(\text { yes }=1, \text { no }=0) \\
\text { A heart attack (including myocardial infarction or coronary thrombosis) }(\text { yes }=1, \text { no }=0) \\
\text { Congestive heart failure }(\text { yes }=1, \text { no }=0) \\
\text { A heart murmur (yes }=1, \text { no }=0) \\
\text { An abnormal heart rhythm }(\text { yes }=1, \text { no }=0) \\
\text { Diabetes or high blood sugar }(\text { yes }=1, \text { no }=0) \\
\text { A stroke (cerebrovascular disease) }(\text { yes }=1, \text { no }=0) \\
\text { Chronic lung disease, such as chronic bronchitis or emphysema }(\text { yes }=1, \text { no }=0) \\
\text { Arthritis (including osteoarthritis or rheumatism) }(\text { yes }=1, \text { no }=0) \\
\text { Osteoporosis, sometimes called thin or brittle bones }(\text { yes }=1, \text { no }=0) \\
\text { Cancer or a malignant tumour }(\text { excluding minor skin cancers) }(\text { yes }=1, \text { no }=0) \\
\text { Parkinson disease (yes }=1, \text { no }=0) \\
\text { Any emotional, nervous or psychiatric problems (yes }=1, \text { no }=0) \\
\text { Alzheimer disease (yes }=1 \text {, no }=0) \\
\text { Dementia, organic brain syndrome, senility or other serious memory problem }(\text { yes }=1, \text { no }=0 \text { ) }\end{array}$ \\
\hline
\end{tabular}




\begin{tabular}{ll}
\hline Type of deficit & Deficit \\
\hline Eyesight & Excellent $=0$, very good $=0.25$, good $=0.5$, fair $=0.75$, poor or blind $=1$ \\
Hearing & Excellent $=0$, very good $=0.25$, good $=0.5$, fair $=0.75$, poor $=1$ \\
Falls & Fallen in the last year $($ yes $=1$, no $=0)$ \\
Hip fracture & Yes $=1$, no $=0$ \\
Joint replaced & Yes $=1$, no $=0$ \\
Correct day of month given & Yes $=0$, no $=1$ \\
Correct year given & Yes $=0$, no $=1$ \\
Correct month given & Yes $=0$, no $=1$ \\
Cognitive function & Total score on composite measure, divided into quartiles $(1$ lowest scores $]=1,2=0.6,3=$ \\
& $0.3,4$ [highest scores $=0)$ \\
Often troubled by severe pain & Often troubled by severe pain $($ yes $=1$, no $=0)$ \\
Pain while walking & Yes $=1$, no $=0$ \\
Incontinence & Lost any urine beyond your control in last 12 months $($ yes $=1$, no $=0)$ \\
Self-rated health & Excellent $=0$, very good $=0.25$, good $=0.5$, fair $=0.75$, poor $=1$ \\
\hline
\end{tabular}

\section{References}

1 Wurm S, Benyamini Y: Optimism buffers the detrimental effect of negative self-perceptions of ageing on physical and mental health. Psychol Health 2014;29:832-848.

$\checkmark 2$ Freeman AT, Santini ZI, Tyrovolas S, Rummel-Kluge C, Haro JM, Koyanagi A: Negative perceptions of ageing predict the onset and persistence of depression and anxiety: findings from a prospective analysis of the Irish Longitudinal Study on Ageing (TILDA). J Affect Disord 2016;199:132-138.

-3 Robertson DA, King-Kallimanis BL, Kenny RA: Negative perceptions of aging predict longitudinal decline in cognitive function. Psychol Aging 2016;31:71-81.

4 Robertson DA, Savva GM, King-Kallimanis BL, Kenny RA: Negative perceptions of aging and decline in walking speed: a self-fulfilling prophecy. PLoS One 2015;10:e0123260.

-5 Sargent-Cox KA, Anstey KJ, Luszcz MA: The relationship between change in self-perceptions of aging and physical functioning in older adults. Psychol Aging 2012;27:750760.

6 Levy BR, Slade MD, Kasl SV: Longitudinal benefit of positive self-perceptions of aging on functional health. J Gerontol B Psychol Sci Soc Sci 2002;57:P409-P417.

7 Beyer AK, Wolff JK, Warner LM, Schuz B, Wurm S: The role of physical activity in the relationship between self-perceptions of ageing and self-rated health in older adults. Psychol Health 2015;30:671-685.

-8 Moser C, Spagnoli J, Santos-Eggimann B: Self-perception of aging and vulnerability to adverse outcomes at the age of 65-70 years. J Gerontol B Psychol Sci Soc Sci 2011;66: 675-680.

-9 Sargent-Cox KA, Anstey KJ, Luszcz MA: Longitudinal change of self-perceptions of aging and mortality. J Gerontol B Psychol Sci Soc Sci 2014;69:168-173.
10 Levy BR, Ferrucci L, Zonderman AB, Slade MD, Troncoso J, Resnick SM: A culturebrain link: negative age stereotypes predict Alzheimer's disease biomarkers. Psychol Aging 2016;31:82-88.

11 Fried LP, Tangen CM, Walston J, Newman AB, Hirsch C, Gottdiener J, Seeman T, Tracy R, Kop WJ, Burke G, McBurnie MA: Frailty in older adults: evidence for a phenotype. J Gerontol A Biol Sci Med Sci 2001;56:M146M156.

12 Rockwood K, Mitnitski A: Frailty in relation to the accumulation of deficits. J Gerontol A Biol Sci Med Sci 2007;62:722-727.

13 Gale CR, Cooper C, Deary IJ, Aihie Sayer A: Psychological well-being and incident frailty in men and women: the English Longitudinal Study of Ageing. Psychol Med 2014;44: 697-706.

14 Vaughan L, Corbin AL, Goveas JS: Depression and frailty in later life: a systematic review. Clin Interv Aging 2015;10:1947-1958.

15 Gale CR, Ritchie SJ, Cooper C, Starr JM, Deary IJ: Cognitive ability in late life and onset of physical frailty: the Lothian Birth Cohort 1936. J Am Geriatr Soc 2017, Epub ahead of print.

16 Rao SK, Wallace LM, Theou O, Rockwood K: Is it better to be happy or not depressed? Depression mediates the effect of psychological well-being on adverse health outcomes in older adults. Int J Geriatr Psychiatry 2016, Epub ahead of print.

17 Steffick DE; HRS Working Group: Documentation of Affective Functioning Measures in the Health and Retirement Study. HRS/ AHEAD Documentation Report DR-005. Ann Arbor, MI, Survey Research Center, University of Michigan, 2000. http://hrsonline. isr.umich.edu/sitedocs/userg/dr-005.pdf.
18 Bandeen-Roche K, Xue QL, Ferrucci L, Walston J, Guralnik JM, Chaves P, Zeger SL, Fried LP: Phenotype of frailty: characterization in the women's health and aging studies. J Gerontol A Biol Sci Med Sci 2006;61:262266.

19 Searle SD, Mitnitski A, Gahbauer EA, Gill TM, Rockwood K: A standard procedure for creating a frailty index. BMC Geriatrics 2008;8:24.

20 Banks J, Karlsen S, Oldfield Z: Socio-economic position; in Marmot M, Banks J, Blundell R, Lessof C, Nazroo J (eds): Health, Wealth and Lifestyles of the Older Population in England. London, Institute of Fiscal Studies, 2003, pp 71-125.

21 Garson G: Missing Values Analysis and Data Imputation. Asheboro, NC, Statistical Associates Publishers, 2015.

22 Graham JW, Olchowski AE, Gilreath TD: How many imputations are really needed? Some practical clarifications of multiple imputation theory. Prev Sci 2007;8:206-213.

23 Levy BR, Pilver C, Chung PH, Slade MD: Subliminal strengthening: improving older individuals' physical function over time with an implicit-age-stereotype intervention. Psychol Sci 2014;25:2127-2135.

24 Morley JE, Vellas B, van Kan GA, Anker SD, Bauer JM, Bernabei R, Cesari M, Chumlea WC, Doehner W, Evans J, Fried LP, Guralnik JM, Katz PR, Malmstrom TK, McCarter RJ, Gutierrez Robledo LM, Rockwood K, von Haehling S, Vandewoude MF, Walston J: Frailty consensus: a call to action. J Am Med Dir Assoc 2013;14:392-397.

25 Rockwood K, Andrew M, Mitnitski A: A comparison of two approaches to measuring frailty in elderly people. J Gerontol A Biol Sci Med Sci 2007;62:738-743. 
26 Sarkisian CA, Prohaska TR, Wong MD, 28 Savela SL, Koistinen P, Stenholm S, Tilvis RS, Hirsch S, Mangione CM: The relationship between expectations for aging and physical activity among older adults. J Gen Intern Med 2005;20:911-915.

27 Levy BR, Myers LM: Preventive health behaviors influenced by self-perceptions of aging. Prev Med 2004;39:625-629.
Strandberg AY, Pitkala KH, Salomaa VV, Strandberg TE: Leisure-time physical activity in midlife is related to old age frailty. J Gerontol A Biol Sci Med Sci 2013;68:14331438.

29 Laidlaw K, Power MJ, Schmidt S, Group W-O: The Attitudes to Ageing Questionnaire (AAQ): development and psychometric properties. Int J Geriatr Psychiatry 2007; 22:367-379.
0 Barker M, O’Hanlon A, McGee HM, Hickey A, Conroy RM: Cross-sectional validation of the Aging Perceptions Questionnaire: a multidimensional instrument for assessing selfperceptions of aging. BMC Geriatr 2007;7:9.

31 North MS, Fiske ST: Modern attitudes toward older adults in the aging world: a crosscultural meta-analysis. Psychol Bull 2015; 141:993-1021. 